\title{
Triple Deuterium Fusion By Three Body Recombination Between Deuteron And The Nuclei Of Lattice Trapped Deuterium Molecules
}

\author{
K. C. ENGVILD \\ PRD 313, Risø National Laboratory, \\ DK-4000 Roskilde, \\ Denmark \\ E-mail:kjeld.engvild@risoe.dk \\ L. KOWALSKI \\ Department of Mathematical Sciences, \\ Montclair State University, \\ Upper Montclair, \\ N. J. 07043, US A \\ E-mail:kowalski@mail.montclair.edu
}

\begin{abstract}
A hypothesis is proposed where low energy nuclear reactions involve three-body recombination of deuterons injected between the nuclei of $\mathrm{D}_{2}$ molecules trapped in a dense lattice of a chemical compound of transition metal and impurity. Two D's fuse to ${ }^{4} \mathrm{He}$, and the energy is converted by expulsion of the third deuteron. Measurable fusion occurs when two D's are confined within 0.1 angstrom of each other. Three boson (efimov) interactions can have longer range than two boson interactions. The best known example is triple alpha fusion to carbon- 12 in stars. Triple deuterium interaction could perhaps be possible in the 0.5-1.0 angstrom range; the distance between D's in a $\mathrm{D}_{2}$ molecule are 0.74 angstrom. The hypothesis accounts for the low reproducibility and short duration of the effect because of rapid destruction of the active structure by sputtering, radiation damage, bubble formation and chemical reduction of the impurities to compounds like $\mathrm{D}_{2} \mathrm{O}, \mathrm{ND}_{3}, \mathrm{CD}_{4}$, or $\mathrm{BD}_{3}$. The hypothesis also accounts for the observed prevalence of ${ }^{4} \mathrm{He}>>$ tritium $>>$ neutrons.
\end{abstract}

\section{Introduction}

Triple deuterium fusion as an explanation for fusion in condensed matter was proposed by Takahashi in $1989^{1,2}$, by Kasagi et al. ${ }^{3}$, Engvild ${ }^{4}$ and several others, The present hypothesis involves injecting a deuteron between the two deuterium nuclei of a deuterium molecule which is trapped in a dense lattice, probably a chemical compound of palladium/titanium-like metal and impurity:

$$
\begin{aligned}
\left.\mathrm{D} \rightarrow\right|_{\mathrm{D}} ^{\mathrm{D}} \rightarrow(3 \mathrm{D}) & \rightarrow{ }^{4} \mathrm{He}+\mathrm{D}(23.8 \mathrm{MeV}) \\
& \rightarrow{ }^{3} \mathrm{He}+\mathrm{T}(9.5 \mathrm{MeV})
\end{aligned}
$$

Triple deuterium fusion has been dismissed by most physicists ${ }^{5}$ because the D's in a normal palladium lattice are almost 3 angstrom apart. The D's are kept separate by their electric charge which is only lightly screened by the electrons of the metal.

If it were possible to keep two D's confined within 0.1 angstrom of each other for a "long" time ${ }^{6}$ the fusion rate would be about one million per second per mole. This separation would be about 10000 times the range of the strong nuclear force, but only about $1 / 8^{\text {th }}$ of the 
distance between the D's of a deuterium molecule (0.74 angstrom). At 0.2 angstrom separation the fusion rate is negligible.

If we had a material where D's could be forced closer together than they are in palladiumlike metals or $\mathrm{D}_{2}$ we might be able to accomplish fusion. Such a material might be titanium or zirconium carbide or nitride (or PdO?, PdB?). These are very dense sodium chloride type lattices with occasional vacancies (minus $\mathrm{C}$ or minus $\mathrm{N}$ ) They might be able to trap deuterium molecules, as it is known that they dissolve more hydrogen than do the free metals ${ }^{7}$. If deuterons were injected between the D's of the deuterium molecules the average separation between the three D's could be less than 0.6 angstrom. In the lattice there is not enough room to form a $\mathrm{D}_{3}{ }^{+}$ion or a $\mathrm{D}_{3}$ Rydberg molecule (Fig. 1)

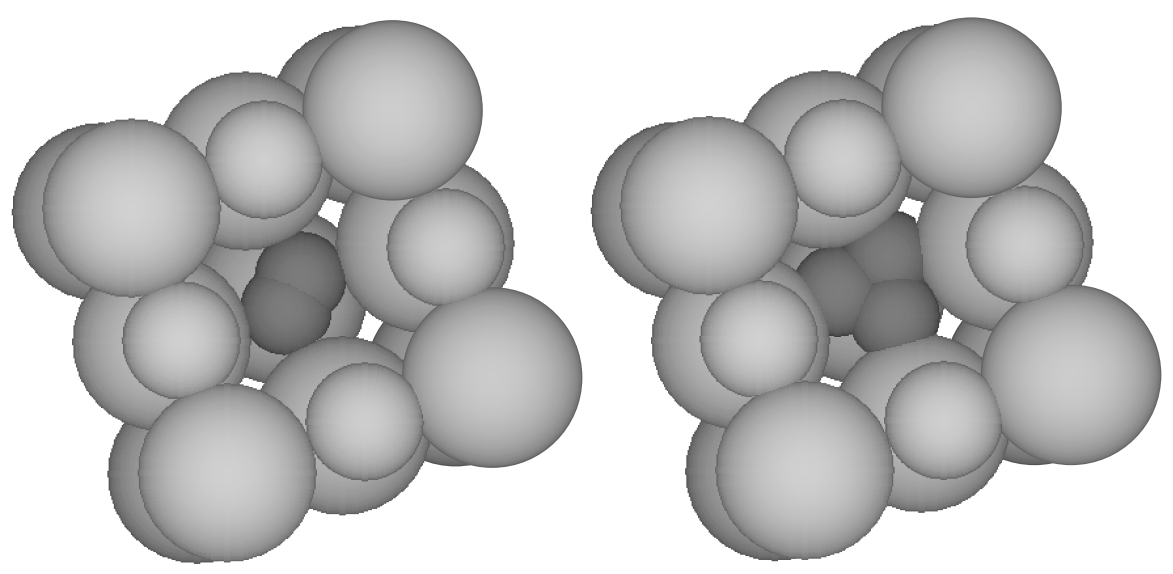

Figure 1. Left: $\mathrm{A}_{2}$ molecule embedded in a vacancy in a zirconium nitride lattice. Right: Not enough space for a $\mathrm{D}_{3}{ }^{+}$ion. The three $\mathrm{D}$ atoms/nuclei squeezed together causing deuterium fusion by three-body recombination.

The triple D complex with an average separation between the D's of less than 0.6 angstrom could possibly turn into a virtual nuclear efimov resonance ${ }^{8,9,10}$. The efimov effect occurs when a system of three particles interact through short range two body potentials and there are two particle bound states very close to zero energy. Efimov resonances are exceedingly outspread and have very low energy. However, there are no known near-zero energy states of DD nuclei; they will tend to collapse into ${ }^{4} \mathrm{He}$ and the energy could be converted by expulsion of the third deuteron.

\section{Active material requirements}

The active material must be a conductor incorporating single $\mathrm{D}_{2}$ molecules sitting alone, tightly bound, preferably not free to rotate. Most such materials are probably chemical compounds, because metals incorporate hydrogen singly in the lattice. The material must not be susceptible to void and bubble formation ${ }^{11}$, where deuterium can exist as a gas, and deuteron injection would only result in the formation of $\mathrm{D}_{3}$ ions. The material must not be susceptible to chemical reduction by $\mathrm{D}$ e. g. the formation of $\mathrm{CD}_{4}, \mathrm{D}_{2} \mathrm{O}, \mathrm{ND}_{3}$ or $\mathrm{BD}_{3}$. The material must be able to withstand sputtering and other radiation damage.

Possible materials could be:

- Silicon, which is known to incorporate hydrogen molecules ${ }^{12}$ although only in low concentrations. A problem is its very low conductivity. 
- $\mathrm{ZrN}, \mathrm{ZrC}, \mathrm{TiN}, \mathrm{TiC}(\mathrm{PdO}, \mathrm{PdB}$ ?) or other of the refractory transition metal compounds with sodium chloride structure. These compounds have very dense lattices with occasional vacancies, which could trap deuterium molecules.

\section{Predictions}

On the basis of the hypothesis it is possible to draw the following consequences: some of these have already been stated by Takahashi ${ }^{1,2}$; notably the relative prevalence of ${ }^{4} \mathrm{He},{ }^{3} \mathrm{He}$, tritium and neutrons.

- Triple deuterium fusion requires "dirty" conditions; most active materials are chemical compounds, not the pure metal.

- Triple D fusion begins after a long lag, where $\mathrm{D}_{2}$ molecules are imbedded in the material.

- Triple D fusion stops again when more D incorporation destroys the active structure by formation of bubbles and voids, by sputtering, or by reduction of vital parts of the structure, e. g. to $\mathrm{D}_{2} \mathrm{O}$ or $\mathrm{ND}_{3}$.

- Helium-4 will by far exceed helium-3 and tritium; an asymmetric approach of the incoming deuteron will be much more likely than a symmetric approach.

- Tritium formation will by far exceed neutron formation. Neutrons will form mainly in secondary reactions with accelerated deuterons, tritiums and alphas.

- A number of radioactive and stable isotopes will be formed after nuclear reactions between elements in the electrode and accelerated $\mathrm{D}, \mathrm{T},{ }^{3} \mathrm{He}$ and ${ }^{4} \mathrm{He}$.

- Gamma rays will result from some of the radioactive isotopes as well as knockout of inner electrons by accelerated nuclei.

- X-rays are caused by a "bremsstrahlung"-like mechanism.

Some other consequences of the hypothesis may occur, if certain conditions apply. If the hypothetical (3D) efimov resonances are so spatially extended that they can knock directly on the surrounding atoms, the energy could be dissipated "mechanically" to the lattice and perhaps cause local ( Kamada $^{13}$ ) melting. This would also have as a result that the energy of the particles may not follow from the nuclear reaction schemes if the energy is divided in unknown ways between the lattice and the particles. Another possible result of a very extended efimov resonance might be that a deuteron is brought very close to a nucleus in the lattice with the resultant formation of other isotopes according to the scheme:

${ }^{\mathrm{a}} \mathrm{Me}+(3 \mathrm{D}) \rightarrow\left({ }^{\mathrm{a}} \mathrm{MeD}\right)+(2 \mathrm{D}) \rightarrow{ }^{\mathrm{a}+2} \mathrm{Me}+$ alpha

other schemes might also be possible. However, this only applies if a nuclear efimov resonance is larger than several angstroms.

\section{Discussion and Experimental Tests}

The major difficulty in condensed matter fusion research has been the ephemeral nature of the effect. This is commented upon in most review papers ${ }^{14,15}$ and is the major reason that main stream physics ${ }^{5}$ does not recognize the effect as a real phenomenon. Some samples of palladium work, others do not. The first crude tests work, later careful experiments with highly purified reagents and careful cleaning fail. The present hypothesis explains these difficulties with the very specific requirement for trapped $\mathrm{D}_{2}$ molecules in a dense lattice. These conditions arise only occasionally during electrolysis or glow discharge experiments. 
Also the often repeated statement that the effect occurs after a long lag, goes through an optimum, and then disappears again ${ }^{14,15,16}$, gets a natural explanation with the hypothesis.

The efimov effect has been much discussed in recent years ${ }^{8,9,10}$. So far nobody has observed a genuine efimov state in nature, and many people believe it is only a mathematical "pathology". However, there are certain nuclear phenomena, that look very much like efimov resonances, even if they are not strictly speaking the real thing.

The most important example is the triple alpha fusion state which is involved in carbon12 formation in stars. Both the ground state and the excited state can be modeled as composed of three alphas ${ }^{10,17}$. The second unbound resonance at $7.6 \mathrm{MeV}$ is much larger than the ground state $1^{10,17}$.

Another example is the occurrence of borromean nuclei ${ }^{10}$, like helium- 6 which consists of alpha $+n+n$. None of the subsystems alpha $+n$ and $n+n$ are bound, but 6-helium is bound. It is very large with an extended neutron halo surrounding the alpha.

Experimental tests of the hypothesis are probably best done by Cecil-Kasagi ${ }^{3,18}$ Takahashi $^{2}$ type experiments with bombardment of a suitable target with rather low energy deuterons. The extensive enhancement of DD fusion in PdO observed by Kasagi et al. ${ }^{18}$ could perhaps be explained by the present hypothesis. Other targets could be refractory carbides and nitrides and silicon, which is known to incorporate $\mathrm{D}_{2}$ as molecule like complexes in the lattice.

Also Karabut ${ }^{16}{ }^{-}$-Claytor ${ }^{19}$-Romodanov type glow discharge experiments in deuterium gas with suitable electrodes of silicon or refractory metal carbides or nitrides could be done. These experiments should involve efficient detection of tritium and helium-4 and investigation of the electrodes for radioactive isotopes. Such experiments could most easily be modified to extract the energy from the reactions, using a glow discharge setup as a "cold fusion water heater".

Electrolysis in $\mathrm{D}_{2} \mathrm{O}$ with electrodes of refractory transition metal compounds are probably the least suited of the possible tests. They do not require quite as sophisticated equipment as glow discharge experiments do.

\section{References}

1. A. Takahashi, J. Nucl. Sci. Technol. 26, 558 (1989).

2. A. Takahashi, K. Maruta, K. Ochiai and H. Miyamaru, Phys Lett. A255, 89 (1999).

3. J. Kasagi, T. Ohtsuki, K. Ishii and M. Hiraga, J. Phys. Soc. Jpn. 64, 777 (1995).

4. K. C. Engvild, Fusion Technol. 34, 253 (1998).

5. J. R. Huizenga, Cold fusion: The scientific fiasco of the century (1993).

6. W. N. Cottingham and D. A. Greenwood, J. Phys. G: Nucl. Part. Phys. 15, L157 (1989).

7. S. Yamanaka and M. Miyake, J. Nucl. Mater. 201, 134 (1993).

8. V. N. Efimov, Sov. J. Nucl. Phys. 12, 589 (1971).

9. D. V. Fedorov and A. S. Jensen, Phys. Rev. Lett. 71, 4103 (1993).

10. E. Nielsen, D. V. Fedorov, A. S. Jensen and E. Garrido, Phys. Reports. 347, 373 (2001).

11. J. B. Condon and T. Schober, J. Nucl. Mater. 207, 1 (1993).

12. E. E. Chen, M. Stavola, W. B. Fowler and J. A. Zhou, Phys. Rev. Lett. 88, 245503 (2002)

13. K. Kamada, H. Kinoshita and H. Takahashi, Jpn. J. Appl. Phys. 35, 738 (1996). 
14. E. Storms, Cold fusion: an objective assessment. (2001). (http://lenrcanr.org/LibFrame1.html)

15. D. J. Nagel, Radiat. Phys. Chem. 51, 653 (1998)

16. A. B. Karabut, Ya. R. Kucherov and I. B. Savvatimova, Phys. Lett. A170, 265 (1992).

17. D. V. Fedorov and A. S. Jensen, Phys. Lett. B389, 631 (1996).

18. J. Kasagi, H. Yuki, T. Baba, T. Noda, T. Ohtsuki and A. G. Lipson, J. Phys. Soc. Jpn. 71, 2281 (2002).

19. T. N. Claytor, D. D. Jackson and D. G. Tuggle, LAUR 95-2687, (http://www.nde.lanl.gov/cf/tritweb.htm), Los Alamos (1996). 